\title{
PRÁTICAS INCLUSIVAS EM CONTEXTO: AÇÕES DE COMUNICAÇÃO ACESSÍVEL EM PORTUGAL E NO BRASIL
}

\author{
PRACTICAS INCLUSIVAS EN CONTEXTO: ACCIONES DE COMUNICACIÓN \\ ACCESIBLES EN PORTUGAL Y BRASIL
}

\section{INCLUSIVE PRACTICES IN CONTEXT: ACCESSIBLE COMMUNICATION ACTIONS IN PORTUGAL AND BRAZIL}

\author{
Eduardo CARDOSO ${ }^{1}$ \\ Alessandra Lopes de Oliveira CASTELINI ${ }^{2}$ \\ Célia Maria Adão de Oliveira Aguiar SOUSA ${ }^{3}$
}

RESUMO: Este artigo objetiva discutir práticas inclusivas de comunicação acessível quanto à orientação e à prevenção de cuidados com a saúde no contexto da pandemia de COVID-19, realizadas em projetos que acontecem em Portugal e no Brasil. As práticas compreendem a produção de pranchas de comunicação acessível e de materiais educativos para educação em saúde com o emprego da Comunicação Aumentativa e Alternativa (CAA), a partir de princípios do Desenho Universal para a Aprendizagem (DUA). Tais práticas, desenvolvidas em universidades conveniadas de ambos os países, articularam estudos e pesquisadores de diferentes áreas, viabilizando a criação de produtos inclusivos, tornando mais acessível a comunicação e favorecendo os cuidados referentes à saúde em tempos de pandemia, enquanto direito de todos. A pesquisa, de cunho qualitativo, ancora-se metodologicamente em revisão narrativa de literatura. A discussão dos resultados parte de revisão bibliográfica, de legislação e de uma reflexão sobre as práticas realizadas. Consideram-se as ações educativas inovadoras ao ampliar o acesso aos informativos de enfrentamento à COVID-19, ressignificando saberes compartilhados e novas formas de aprendizagens.

PALAVRAS-CHAVE: Educação inclusiva. Práticas pedagógicas. Comunicação acessível. Comunicação aumentativa e alternativa. Desenho universal para a aprendizagem.

RESUMEN: Este artículo tiene como objetivo discutir prácticas inclusivas de comunicación accesible en materia de orientación y prevención de la atención de la salud en el contexto de la pandemia COVID-19, realizadas en proyectos que se desarrollan en Portugal y Brasil. Las prácticas comprenden la producción de tableros de comunicación accesibles y materiales educativos para la educación en salud con el uso de la Comunicación Aumentativa y

${ }^{1}$ Universidade Federal do Rio Grande do Sul (UFRGS), Porto Alegre - RS - Brasil. Professor do Programa de Pós-Graduação em Design e do Departamento de Design e Expressão Gráfica da Faculdade de Arquitetura. Doutorado em Design (UFRGS). ORCID: https://orcid.org/0000-0002-1202-1779. E-mail: eduardo.cardoso@ufrgs.br

${ }^{2}$ Universidade Federal do Piauí (UFPI), Picos - PI - Brasil. Docente e Pesquisadora da Coordenação de Pedagogia/CSHNB. Doutorado em Diversidade Cultural e Inclusão Social (FEEVALE). ORCID: https://orcid.org/0000-0002-8113-5577.E-mail: alessandralopes@ufpi.edu.br

${ }^{3}$ Instituto Politécnico de Leiria (IPL), Leiria - Portugal. Investigadora Integrada do Centro Interdisciplinar de Ciências Sociais, Polo do IPLeiria (CICS.NOVA. IPLeiria). Doutorado em Formação de Professores (UNEX) Espanha. ORCID: https://orcid.org/0000-0002-5113-9323.E-mail: celia.sousa@ipleiria.pt

RIAEE - Revista Ibero-Americana de Estudos em Educação, Araraquara, v. 16, n. esp. 4, p. 2990-3004, dez. 2021. e-ISSN: 1982-5587 
Alternativa (CAA) basada en los principios del Diseño Universal para el Aprendizaje (DUA). Dichas prácticas, desarrolladas en universidades acreditadas de ambos países, articularon estudios e investigadores de diferentes áreas, posibilitando la creación de productos inclusivos, haciendo más accesible la comunicación y favoreciendo la atención de la salud en tiempos de pandemia, como un derecho de todos. La investigación, de carácter cualitativo, está anclada metodológicamente en una revisión narrativa de la literatura. La discusión de los resultados comienza con una revisión bibliográfica, legislación y una reflexión sobre las prácticas realizadas. Se plantean acciones educativas innovadoras para ampliar el acceso a la información sobre la lucha contra el COVID-19, dando un nuevo significado al conocimiento compartido y nuevas formas de aprendizaje.

PALABRAS CLAVE: Educación inclusiva. Prácticas pedagógicas. Comunicación accesible. Comunicación aumentativa y alternativa. Diseño universal para el aprendizaje.

ABSTRACT: This article aims to discuss inclusive practices of accessible communication regarding the guidance and prevention of health care in the context of the COVID-19 pandemic, carried out in projects that take place in Portugal and Brazil. The practices comprise the production of accessible communication boards and educational materials for health education with the use of Augmentative and Alternative Communication (AAC) based on the principles of Universal Design for Learning (UDL). Such practices, developed in accredited universities in both countries, articulated studies and researchers from different areas, enabling the creation of inclusive products, making communication more accessible and favoring health care in times of pandemic, as a right for all. The research, of a qualitative nature, is methodologically anchored in a narrative literature review. The discussion of the results starts with a bibliographical review, legislation and a reflection on the practices carried out. Innovative educational actions are considered to expand access to information on the fight against COVID-19, giving new meaning to shared knowledge and new forms of learning.

KEYWORDS: Inclusive education. Pedagogical practices. Accessible communication. Augmentative and alternative communication. Universal design for learning.

\section{Introdução}

O ano de 2020 colocou o processo educacional em uma nova ordem, impondo muitos desafios aos educadores. O contexto da pandemia mundial de Covid-19 alterou, de forma significativa, o modo de se pensar e de se fazer a educação formal, da Educação Infantil ao Ensino Superior, bem como mudou, repentinamente, o modo de vida da população, exigindo formas de comunicação mais acessíveis, estimulando respostas do campo científico a eventos dessa natureza, gerando intensa mobilização social com foco em ações de prevenção e de enfrentamento do novo coronavírus: SARS-COV-2.

$\mathrm{O}$ enfrentamento da situação pandêmica instituiu, como meta primeira, o afastamento social iminente, que decorreu por todo o ano letivo e avançou em 2021, deslocando crianças e 
profissionais ao ambiente doméstico, repercutindo na reorganização familiar e profissional sem precedentes, tendo como cenário um contexto social permeado por dúvida, medo e insegurança, no entanto potente de novas estratégias, que necessitam ser cada vez mais acessíveis a todos.

A partir da perspectiva expressa no Art. 26 da Declaração Universal dos Direitos Humanos (ONU, 1948), ou seja, de que todo ser humano tem direito à instrução, lideranças mundiais articuladas às orientações da Organização Mundial da Saúde (OMS) buscaram, por meio da comunicação, investir em campanhas, a fim de levar a informação através de diferentes plataformas, enfatizando a importância de instruções e de medidas básicas de higiene e de prevenção, viabilizando práticas pedagógicas inclusivas, apoiadas em recursos e em tecnologias, possibilitando práticas de comunicação mais acessíveis (OMS, 2020).

Ao refletir sobre as repercussões das medidas preventivas no período pandêmico, buscou-se por soluções inclusivas, a partir da comunicação sem barreiras, ampliando a acessibilidade às orientações em saúde. Nessa perspectiva, este estudo socializa atividades realizadas em dois laboratórios de universidades conveniadas no contexto tanto de Portugal, quanto do Brasil, com o auxílio de práticas pedagógicas inclusivas, que passaram a ser desenvolvidas em ambos os grupos, considerando o momento atual. Tais feitos mobilizaram resposta imediata e efetiva da população, haja vista a demanda urgente por estratégias diferenciadas e inovadoras quanto às formas de comunicar, tornando as práticas pedagógicas mais inclusivas e acessíveis.

Sobre o desenvolvimento de produtos e de ações educativas mais inclusivas, destacarse-á a utilização de estratégias apoiadas na Comunicação Aumentativa e Alternativa (CAA), que, conforme Chun (2009), desfrutam de recursos tecnológicos e de técnicas que viabilizam formas de comunicar nas Tecnologias Assistivas (TA), as quais, segundo Bersch (2009), são produtos ou serviços que permitem e promovem a participação plena das pessoas.

Aliados à combinação desses recursos, desenvolveram-se as ações, a partir de princípios do Desenho Universal para a Aprendizagem (DUA), que têm como base atender a todos ao estabelecer múltiplas formas de acesso ao conteúdo, possibilitando, dessa forma, a ampliação do acesso às orientações por contemplar diferentes necessidades para que todos possam aprender, diminuindo, consequentemente, as barreiras que impedem o acesso à informação e à aprendizagem por todos, sem distinção. O DUA visa a proporcionar múltiplos meios de representação - o "que" aprender; de ação e de expressão - o "como" da aprendizagem; e de envolvimento - o "porquê” da aprendizagem (CAST, 2014). 
A necessidade de produções, de adaptações e de soluções em tempos de Covid-19 motivou a aprofundar investigações e a estabelecer apontamentos para o contexto educativo, a partir das seguintes problemáticas: como promover práticas pedagógicas inclusivas com recursos de acessibilidade na comunicação em tempos de pandemia? Quais estratégias da CAA podem ser utilizadas? Como ampliar os processos comunicativos e sensibilizar os profissionais na redução de barreiras na comunicação?

Em Portugal, as ações desenvolveram-se no Centro de Recursos para a Inclusão Digital (CRID), junto ao Instituto Politécnico de Leiria - IPLeiria, que, desde 2006, promove ações inclusivas, compreendendo diversos projetos por meio de protocolos com vários países. Com o avanço da pandemia nos primeiros meses de 2020, houve a necessidade de ampliar tais ações, que repercutiram em soluções específicas de orientações e de educação em saúde mais inclusivas. O projeto intitulado Comunicar sem barreiras, em tempos de Covid-19 iniciou as atividades em 2020 e essas logo repercutiram em ações replicadas no Brasil e em Cabo Verde por meio da Associação Colmeia.

Equipes de profissionais para trabalho remoto foram mobilizadas com o propósito de favorecer o acesso às informações na produção de flyers educativos com orientações de saúde e de prevenção, além de pranchas de comunicação, que foram disponibilizadas de forma gratuita à comunidade. Tais ações implicaram em acessibilidade à informação e à comunicação, oportunizando formas de expressão de necessidades em situações de cuidados com a saúde e nos casos de hospitalização, bem como em orientações educativas sobre regras e protocolos oficiais para reabertura de espaços comerciais e culturais, democratizando o acesso à informação com ações que promovem a cidadania e os cuidados em saúde.

No Brasil, o projeto Pranchas de Comunicação Alternativa em Hospitais, concebido de forma multidisciplinar junto à Universidade Federal do Rio Grande do Sul (UFRGS), articulou ações para prestar auxílio aos profissionais de saúde e aos familiares, durante o atendimento e o acompanhamento de pacientes com dificuldades na comunicação por estarem limitados ou impedidos de realizar a comunicação oral. O projeto contou, ainda, com o apoio da Secretaria Municipal de Desenvolvimento Social e Esporte de Porto Alegre, no Rio Grande do Sul.

Dessa forma, o presente estudo, de cunho qualitativo (MERRIAM, 1998), com processo metodológico ancorado na revisão narrativa de literatura (MARTINS, 2018), buscou mapear conhecimentos sobre os conceitos de CAA e TA, possibilitando atender às demandas emergentes do contexto pandêmico, por meio de práticas pedagógicas inclusivas e inovadoras, que possibilitam a comunicação mais acessível. 
Cordeiro et al. (2007) descrevem que, na revisão narrativa de literatura, não há critério explícito e sistemático para a busca e a análise crítica das evidências, cabendo ao pesquisador decidir quais informações são mais relevantes.

A coleta de dados deu-se por meio da pesquisa bibliográfica e documental e apontou a produção de práticas pedagógicas inclusivas com intervenções nos espaços hospitalares em ambos os países, ocorrendo via disponibilização de materiais educativos e de suporte à comunicação em CAA, que foram liberados em diferentes meios, tais como impressos, em redes sociais e nos sites institucionais, replicados em Portugal e no Brasil.

Ao aprofundar conceitos em relação ao objeto de estudo, a investigação possibilitou conduzir a pesquisa para caminhos significativos quanto ao conhecimento teórico, ampliando discussões necessárias sobre formas de tornar a comunicação mais acessível, socializando ações pedagógicas e inclusivas, favorecendo práticas de educação em saúde, oportunizando benefícios à sociedade.

Este estudo possibilitou considerar que as práticas pedagógicas inclusivas apresentadas favoreceram ações de educação em saúde, enquanto práticas de comunicação acessíveis e inovadoras, ampliando o acesso aos informativos oficiais de enfrentamento à COVID-19, garantindo o direito à comunicação e às orientações de saúde para todos, sem exceção. Ademais, contribuíram para o atendimento e a recuperação de pessoas com necessidades complexas de comunicação em hospitais, havendo mais segurança e conforto.

\section{Comunicação Acessível em tempos de pandemia}

Este trabalho assevera a educação e a saúde enquanto direitos humanos e como direito de todos, conforme a Constituição Federal do Brasil, de 1988 (BRASIL, 1988), assim como o acesso a informações e à disponibilização de recursos de comunicação acessíveis expressos na Lei Brasileira da Inclusão - LBI, Lei n. 13.146/2015 (BRASIL, 2015).

A LBI (BRASIL, 2015) assegura a adoção de medidas e de práticas de higiene, cuidados com a saúde e a proteção às pessoas com deficiência, apoiados em recursos comunicacionais, que necessitam ser inclusivos, refletindo as especificidades existentes nos processos de ensino e de aprendizagem, fomentando, assim, o desenvolvimento de produtos de apoio, que viabilizem a comunicação mais acessível a todos.

Em Portugal, o compromisso com a educação inclusiva corrobora diretrizes da Unesco (2009), enquanto processo que visa responder à diversidade de necessidades por meio do aumento da participação de todos na aprendizagem e ao longo da vida. Com a promulgação 
do Decreto-Lei n. 54/2018 (PORTUGAL, DGE, 2018), estabeleceu-se como uma das prioridades da ação governativa a aposta numa escola inclusiva, a partir da qual todos, independentemente da sua situação pessoal e social, encontram respostas que lhes possibilitam a aquisição de um nível de educação e de formação facilitador da sua plena inclusão social.

Dentre as diversas orientações expressas pela OMS, em tempos de pandemia de COVID-19, destaca-se a importância de viabilizar a comunicação simples e acessível a todos na divulgação de um guia ${ }^{4}$ (OMS, 2020), que disponibilizou orientações para enfrentar as consequências do COVID-19, impulsionando diversos projetos em torno de ações educativas.

Devido às "altas taxas de transmissibilidade e considerável letalidade", conforme estudo desenvolvido por Antônio Augusto Moura da Silva (2020, p.01), deu-se a repercussão em ações pedagógicas e inclusivas, sob caráter de urgência, considerando a proliferação do vírus e do profundo prejuízo econômico e de vidas. Para Santos (2020), a pandemia e a quarentena "estão a revelar que são possíveis alternativas, que as sociedades se adaptam a novos modos de viver quando tal é necessário e sentido como correspondendo ao bem comum" (SANTOS, 2020, p. 29).

A proliferação do coronavírus pela Europa nos primeiros meses de 2020 desencadeou a rápida decisão dos governantes de Portugal pelo isolamento social, proteção da população e busca de soluções tecnológicas e de produtos para viabilizar o atendimento da população em tempo de crise. Posteriormente, no Brasil, ações intensificadas resultaram na adoção de medidas em busca de promover o distanciamento social e de evitar aglomerações, resultando em ações que fecharam as escolas e as universidades, seguidas dos serviços do comércio, resguardando apenas o serviço de saúde e os serviços essenciais à população (DN/PT, 2020).

O isolamento social desencadeou novas formas de enfrentamento perante a situação de alto risco de contágio, para as quais mostrou-se necessário desenvolver novas estratégias e repensar saberes pedagógicos que viabilizassem a situação emergencial e a orientação à população para os devidos cuidados com a saúde física, mental e emocional frente à quarentena, garantindo o direito à comunicação e aos cuidados em saúde na pandemia.

4 Disponível em: https://www.paho.org/es/documentos/consideraciones-psicosociales-salud-mental-durantebrote-covid-19. Acesso em: 10 mar. 2021. 


\section{Apresentação e reflexões sobre os materiais desenvolvidos}

Partindo do pressuposto de que a comunicação é uma das mais importantes necessidades do ser humano, e de que o ato de ler faz parte do processo de comunicação, Célia Sousa (2012) evidencia que essa prática se torna vital para o desenvolvimento das pessoas, visto que ocorre entre os sujeitos de forma cotidiana, envolvendo as interações sociais e as trocas fomentadas por diversas situações.

Dentre os trabalhos desenvolvidos no CRID em Portugal, as ações sobre comunicação acessível são fundamentais. Nesse sentido, destaca-se o desenvolvimento de uma prancha de

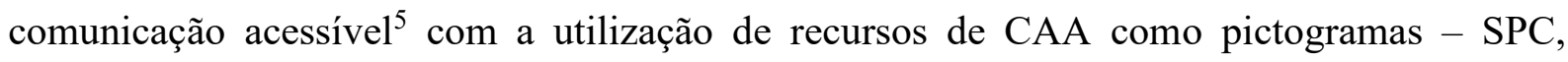
operada por meio do Software Boardmaker ${ }^{\circledR}$ (https://goboardmaker.com/). Essa estratégia viabiliza a adaptação de textos por meio de símbolos e de imagens que facilitam a compressão por diferentes públicos, como pessoas não alfabetizadas, com baixa literacia, estrangeiros que não dominam a língua e por pessoas que apresentam algum tipo de deficiência intelectual e/ou limitações para compreender informações imprescindíveis, nesse momento de pandemia e de ampliação dos cuidados em saúde.

Figura 1 - Prancha Comunicar sem Barreiras, Portugal, 2020

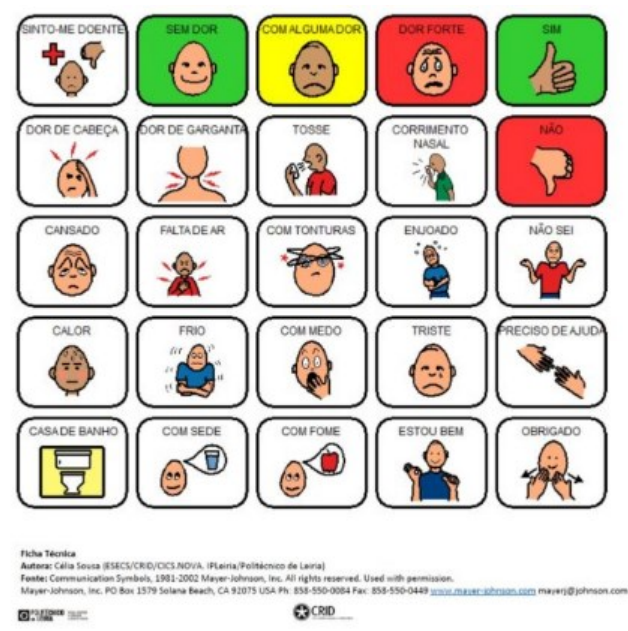

Fonte: Elaborado pelos autores

A utilização da prancha de comunicação acessível, conforme figura 1, sugere que a pessoa aponte para os símbolos ou fixe o olhar para comunicar. A prancha pode ser acessada gratuitamente no site do $\mathrm{CRID}^{6}$, podendo ser utilizada em contexto digital por meio de

${ }^{5}$ Disponível em: https://gazetadascaldas.pt/coronavirus/coronavirus-centro-de-recursos-do-politecnico-de-leiriacria-tabela-para-comunicar-a-saude/. Acesso em: 10 maio 2021.

${ }^{6}$ Disponível em: https://crid.esecs.ipleiria.pt/2020/04/22/crid-apresenta-mensagens-em-pictogramas-em-tempode-pandemia/. Acesso em: 10 jun. 2021.

RIAEE - Revista Ibero-Americana de Estudos em Educação, Araraquara, v. 16, n. esp. 4, p. 2990-3004, dez. 2021. e-ISSN: 1982-5587 
smartphone, tablet ou de forma impressa. Também pode ser plastificada, aumentando assim a durabilidade e permitindo a higienização. O material desenvolvido nos primeiros meses de 2020 foi logo disponibilizado a profissionais da saúde nos processos de intervenção com pacientes hospitalizados e no auxílio a familiares e a pessoas idosas com baixa literacia, que apresentam dificuldades para comunicar oralmente.

Além das pranchas para comunicação, a equipe do CRID passou a desenvolver flyers com informações importantes, a considerar durante o período de isolamento social e os cuidados com a saúde em tempos de pandemia, além de adaptações nas orientações repassadas pelo governo e pela OMS. Dentre os materiais produzidos no período de março a junho de 2020, foram encontrados no site do CRID e nas redes sociais quarenta flyers com diferentes tipos de conteúdo que foram criados e adaptados com SPC.

O material tem possibilitado o acesso por um número considerável de pessoas. Os conteúdos dos materiais foram agrupados em quatro categorias: orientações em saúde; regras para reabertura e protocolos; datas comemorativas e entretenimento.

Quadro 1 - Flyers Acessíveis na Covid-19 em Portugal

\begin{tabular}{|c|c|c|}
\hline CATEGORIAS & CONTEÚDO & TOTAL \\
\hline Saúde & $\begin{array}{l}\text { Informações sobre cuidados com o corpo, uso de máscara, higiene das } \\
\text { mãos, distanciamento social, estado de calamidade. }\end{array}$ & 15 \\
\hline $\begin{array}{c}\text { Regras para } \\
\text { Reabertura e } \\
\text { Protocolos }\end{array}$ & $\begin{array}{l}\text { Normas na abertura dos museus, nos cabeleireiros e barbeiros, para } \\
\text { clientes da restauração e bebidas, atendimento em lojas, regras na praia. }\end{array}$ & 11 \\
\hline $\begin{array}{c}\text { Datas } \\
\text { Comemorativas }\end{array}$ & $\begin{array}{l}\text { Dia do Trabalhador, Dia das Mães, Dia das Crianças e mensagens dos } \\
\text { órgãos do turismo e pastoral dos deficientes. }\end{array}$ & 05 \\
\hline Entretenimento & Poesias, brincadeiras, jogos, canções, danças, receitas. & 09 \\
\hline & Total & 40 \\
\hline
\end{tabular}

Fonte: Elaborado pelos autores

Diante dos dados expostos no Quadro 1, foi possível perceber que os conteúdos disponibilizados, em maior parte, asseguram informações sobre os cuidados em saúde e as regras/normas que orientam as pessoas para reabertura e protocolos de uso de espaços públicos. As peças de entretenimento trataram de conteúdos diversos voltados às crianças e à interação com familiares, oportunizando momentos de diversão e de aprendizagem com práticas educativas, de orientação e modos de brincar que fazem parte do cotidiano das pessoas e consideram o contexto cultural local.

Os flyers contemplam o emprego coordenado de técnicas da CAA como a escrita simples e com pictogramas. A utilização da escrita simples, conforme Martins (2014), consiste em uma forma de tornar o texto objetivo de ler e de compreender, partindo de 
conceitos familiares, sendo útil para todos. Além disso, destaca-se que a utilização de fonte ampliada beneficia as pessoas com baixa visão.

A adaptação da escrita com símbolos pictográficos de comunicação (SPC) busca beneficiar pessoas que ainda não se apropriaram da língua escrita ou pessoas que apresentam alguma dificuldade de comunicação. A utilização de símbolos SPC específicos para os cuidados da saúde e do contexto de Covid-19 facilitam a compreensão e tornam mais significativas as informações apresentadas. Dentre os aspectos evidenciados na pesquisa, observa-se a perspectiva inclusiva na produção dos flyers educativos por meio das estratégias articuladas de CAA e TA, visto que os materiais podem ser baixados em smartphones ou tablets, oportunizando a interação no contexto digital e ampliando o acesso e a aprendizagem por todos.

Sobre a perspectiva inclusiva, a produção dos flyers baseou-se no Universal Designer Learning (UDL), que surgiu nos Estados Unidos em 1999, traduzido para o português como Desenho Universal para a Aprendizagem (DUA), que consiste na elaboração de estratégias para tornar os produtos mais acessíveis por todos, contemplando aspectos físicos, de serviços, de produtos e de soluções educacionais para que todos possam aprender sem barreiras (CAST, 2006).

O DUA consiste em um conjunto de princípios e constitui um modelo prático que objetiva maximizar as oportunidades de aprendizagem para todos. Possui como objetivo auxiliar os educadores e demais profissionais a adotarem modos de aprendizagem adequados, escolhendo e desenvolvendo materiais e métodos eficientes, a fim de ser elaborado de maneira mais aprimorada e acessível para avaliar o progresso de todos os estudantes. Dessa maneira, ao invés de desenvolver uma adaptação específica para um aluno particular, em determinada atividade, opta-se por criar formas diferenciadas de ensinar determinado conteúdo para todos.

A utilização da abordagem DUA (CAST, 2014) mostrou ser eficiente para a criação de ambientes de aprendizagem flexíveis e acessíveis para todos, incluindo aqueles com deficiência (RIBEIRO; AMATO, 2018). Nessa perspectiva, a adoção dessa proposta justificase ao compreender que a utilização de diversos recursos pedagógicos e tecnológicos, bem como materiais, técnicas e estratégias, objetivam atender a diversidade leitora em busca de ampliar o acesso à informação e de facilitar a aprendizagem por todos, independentemente de suas deficiências (CASTELINI; SOUSA; QUARESMA DA SILVA, 2020).

Nesse sentido, os flyers educativos produzidos apresentam-se como suporte para os profissionais da saúde, educadores, familiares, acompanhantes dos pacientes e demais 
envolvidos, oportunizando reflexões sobre a importância da comunicação acessível. Essas reflexões tornam-se relevantes ao pensar o contexto educativo e a formação inicial e continuada de profissionais ao apontar caminhos para a elaboração, adaptação e inovação de produtos inclusivos, enquanto práticas pedagógicas como ferramentas que visam à acessibilidade, seja em termos físicos, criação de produtos ou soluções educacionais, para que todos possam aprender e se comunicar (CAST, 2013).

No Brasil, as atividades desenvolvidas foram realizadas de forma multidisciplinar, conforme citado, e contemplaram a pesquisa e o desenvolvimento de pranchas de CAA com símbolos gráficos específicos para o uso em hospitais e espaços de saúde em tempos de pandemia de Covid-19, auxiliando os pacientes na comunicação de sentimentos e de sensações, além de possibilitar a resposta a questionamentos e de permitir solicitações simples do cotidiano. Para a utilização das pranchas foram disponibilizadas orientações ${ }^{7}$ no site do Grupo COM Acesso ${ }^{8}$, que informa aos profissionais de saúde, familiares e acompanhantes de pacientes a melhor forma de utilização das estratégias de CAA e os modos pelos quais o paciente pode se expressar, seja pelo apontamento manual ou rastreamento ocular dos símbolos e letras nos cartões.

Figura 2 - Pranchas de Comunicação Alternativa, Brasil, 2020
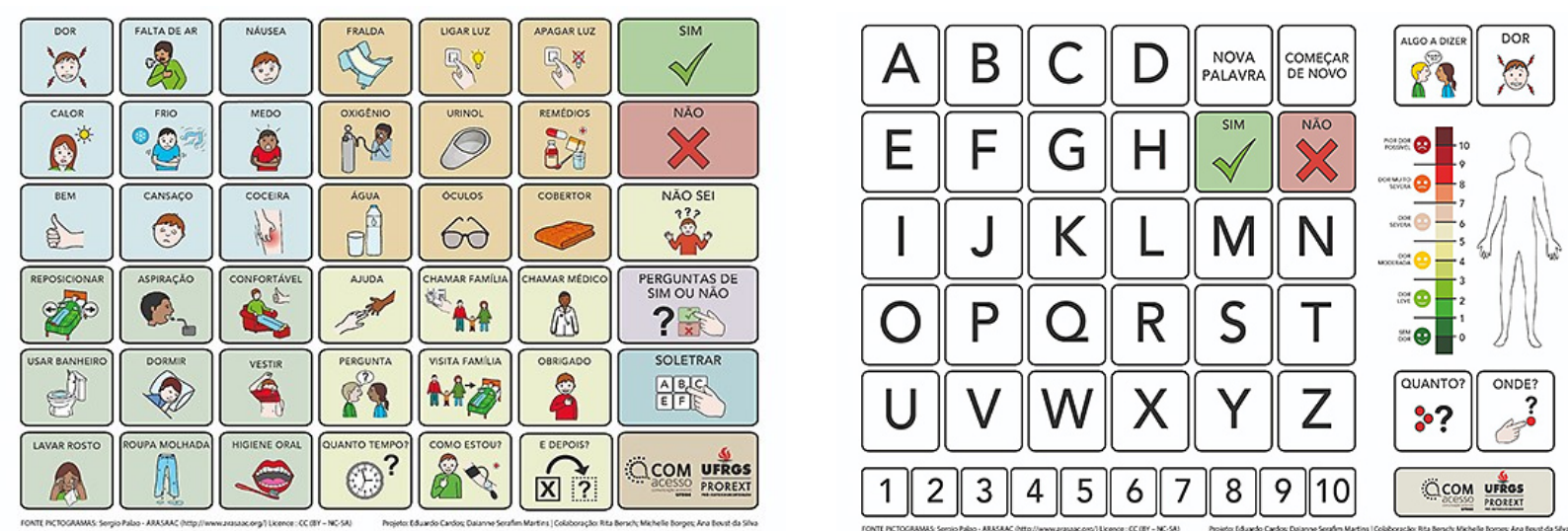

Fonte: Elaborado pelos autores

As pranchas desenvolvidas no projeto da UFRGS apresentam pictogramas e letras organizados por grupos. O material é disponibilizado para download, mas também foi entregue impresso, frente e verso e plastificado, a todos os hospitais de Porto Alegre e,

${ }^{7}$ Disponível em: https://www.ufrgs.br/comacesso/wp-content/uploads/2020/04/INSTRUCOES-pranchahospitalar_V.pdf. Acesso em: 10 maio 2021.

${ }^{8}$ Disponível em: https://www.ufrgs.br/comacesso/pranchas-caa-hospitalar/. Acesso em: 10 jun. 2021. 
posteriormente, aos hospitais do estado do Rio Grande do Sul, totalizando mais de 3000 pranchas entregues de março a dezembro de 2020.

$\mathrm{O}$ alfabeto permite à pessoa soletrar ou apontar, formando palavras e frases simples. Os números disponibilizados auxiliam na elaboração dessas frases e na medição da dor, enquanto técnica frequentemente utilizada por profissionais da saúde. O material, ainda, contempla o desenho do corpo humano com uma escala que permite expressar, por meio de símbolos, os graus de dor ou de desconforto do paciente, auxiliando na verificação de sintomas e no bem-estar da pessoa que está em atendimento de saúde.

A base de pictogramas utilizada neste trabalho parte do Portal Aragonés de la Comunicación Aumentativa y alternativa (Portal ARASAAC; http://www.arasaac.org/), o qual apresenta um conjunto de símbolos, fotografias, números, letras do alfabeto e outros desenhos, tornando possível acrescentar e combinar símbolos. Nesses sistemas, a palavra escrita localiza-se acima de cada pictograma e o tamanho indicado para a sua aplicação é de aproximadamente $3 \mathrm{~cm}$ (VERZONI, 1999). Os pictogramas ainda passaram por adaptações e um redesenho, com vistas à unidade de linguagem em todo o material. Assim, também, criouse uma versão das pranchas em fonte ampliada, com quadrantes maiores para o apontamento e a leitura, para facilitar a utilização em pacientes com Covid-19 nos procedimentos hospitalares.

Por meio de parceria com a ISAAC Brasil - International Society of Alternative and Augmentative Communication - Capítulo Brasileiro, traduziu-se o material para sete idiomas: português de Portugal, Inglês, Espanhol, Alemão, Italiano, Francês e Chinês. Além disso, produziram-se vídeos educativos para a formação geral sobre os conceitos de (1) Tecnologia Assistiva e (2) Comunicação Alternativa, (3) o uso de pranchas de comunicação alternativa e (4) emprego específico no contexto hospitalar. O material também conta com recursos de acessibilidade, como Libras (Língua Brasileira de Sinais) e Legendas para Surdos e Ensurdecidos (LSE). A ISAAC Brasil, igualmente, promoveu o contato com associações e conselhos das áreas de saúde para parceria quanto à distribuição do material, resultando na entrega de mais de três mil pranchas em outros estados do Brasil.

Por fim, o projeto ganhou versão em aplicativo para equipamentos móveis ${ }^{9} \mathrm{e}$ on-line com vistas a facilitar o acesso e a promover a comunicação das pessoas também após

9 Disponível em: https://apps.apple.com/us/app/mobboards/id1565762601. Acesso em: 10 maio 2021.; Disponível em: https://play.google.com/store/apps/details?id=mobfeel.com.br.mobboards.mobboards. Acesso em: 10 maio 2021.; Disponível em: http://mobfeel.com.br/mobboards/. Acesso em: 10 maio 2021.

RIAEE - Revista Ibero-Americana de Estudos em Educação, Araraquara, v. 16, n. esp. 4, p. 2990-3004, dez. 2021. e-ISSN: 1982-5587 
deixarem o ambiente hospitalar, uma vez que as pranchas de comunicação permanecem nos espaços de saúde.

\section{Considerações finais}

As investigações empreendidas neste estudo fortalecem o diálogo interdisciplinar ao evidenciar práticas pedagógicas inclusivas enquanto práticas educativas que favorecem a comunicação acessível e a educação em saúde, desenvolvidas em Portugal e no Brasil no contexto da pandemia de Covid-19.

De acordo com Nunes e Madureira (2015), evidencia-se a urgência em se pensar em recursos, práticas e intervenções mais inclusivas de modo a garantir o acesso e a participação de todos. Nessa perspectiva, a utilização de recursos de acessibilidade na comunicação tornase essencial nos cuidados com a saúde em tempos de pandemia, pois possibilita a interação com profissionais da saúde e com familiares, além de ampliar a participação nos processos educativos, sociais e efetivação da cidadania ativa.

Nesse sentido, é importante salientar que as ações desenvolvidas em ambos os projetos contemplaram a produção de materiais para a comunicação acessível em grupos de universidades de Portugal e do Brasil, que desenvolvem projetos de CAA e TA há alguns anos, considerando o contexto cultural e as especificidades de cada país, procurando manter a unidade estética dos materiais. Os projetos desenvolvidos no contexto da pandemia de Covid19 foram direcionados a essa finalidade, adaptados para o caso, em específico, e auxiliaram na associação de imagens, orientações educativas em saúde e na forma de utilização dos materiais, bem como na disponibilização gratuita nos formatos impresso e digital, dando-se tal ação em ambos os países.

Das estratégias de CAA utilizadas, observou-se a adoção, em ambos os projetos, de estratégias de escrita simples e com símbolos pictográficos de comunicação, embora a partir de diferentes bases de pictogramas. Em Portugal, há o predomínio do uso do SPC por meio do software Boardmaker ${ }^{\circledR}$ com a licença proposta pelo governo. No Brasil, a opção pelo uso de sistemas com licença Creative Commons deve-se pela indisponibilidade de recursos para aquisição e manutenção de sistemas proprietários pelo Governo.

A partir do estudo, constatou-se a ausência de uma normatização da CAA em ambos os países, mas pôde-se observar a existência de um empenho entre os grupos para manter unidade no desenvolvimento dos materiais. Desse modo, a eficácia das pranchas de comunicação e dos flyers produzidos para o período de pandemia de Covid-19 ainda carece de 
pesquisas e de testes de recepção com os usuários, segundo os objetivos e as tecnologias estabelecidos para cada contexto e objetivos de comunicação.

Evidenciou-se, ainda, a relevância do tema em ambos os países, resultando no fortalecimento de ações governamentais e da sociedade na implementação dos produtos inclusivos e nas práticas educativas de orientação de cuidados e de enfrentamento da Covid19. A mobilização proposta pelos projetos em tempos de pandemia possibilitou a construção de diálogos interdisciplinares de diferentes profissionais na elaboração de produtos inclusivos, ampliando as redes de trabalho em prol de acessibilidades na comunicação e práticas educativas em benefício da saúde e na redução de barreiras na aprendizagem.

\section{REFERÊNCIAS}

BERSCH. R. C. R., Design de um serviço de tecnologia assistiva em escolas públicas. 2009. 231 f. Dissertação (Mestrado em Design) - Universidade Federal do Rio Grande do Sul, Porto Alegre, 2009. Disponível em: http://www.lume.ufrgs.br/bitstream/handle/10183/18299/000728187.pdf?. Acesso em: 10 maio 2021

BRASIL. Constituição Federal da República Federativa do Brasil de 1988. Brasília, DF: Presidência da República, 05 out. 1988. Disponível em:

http://www.planalto.gov.br/ccivil_03/constituicao/constituicaocompilado.htm. Acesso em: 10 maio 2021.

BRASIL. Lei n. 13.146, de 6 de julho de 2015. Institui a Lei Brasileira de Inclusão da Pessoa com Deficiência (Estatuto da Pessoa com Deficiência). Brasília, DF, 2015. Disponível em: http://www.planalto.gov.br/ccivil_03/_ato2015-2018/2015/lei/113146.htm. Acesso em: 10 maio 2021.

CAST UDL. Learn About Universal Design for Learning (UDL). 2006. Disponível em: http://bookbuilder.cast.org/learn.php. Acesso em: 10 maio 2021.

CAST, UDL. Book Builder. Massachusetts Department of Elementary \& Secondary Education, NEC Foundation of America. The John W. Alden Trust, and the Pinkerton Foundation. 2013. Disponível em: http://bookbuilder.cast.org/. Acesso em: 10 maio 2021.

CAST, UDL. Center for Applied Special Technology. 2014. Disponível em: http://www.cast.org/udl/index.html. Acesso em fev. 2020.

CASTELINI. A. L. O; SOUSA, C.; QUARESMA DA SILVA, D. R. Tecendo Saberes na Formação Docente: Ações desenvolvidas no Centro de Recursos para Inclusão Digital para a promoção da Comunicação mais Acessível à Todos. In: CONFERÈNCIA INTERNACIONAL DE RECERCA EN EDUCACIÓ, 1., 2020, Barcelona. Anais [...]. Barcelona, Espanha: Universitat de Barcelona; Albacete: LiberLibro, 2020. v. 1, p. 749-760. ISBN: 978-84-17934-76-7. 
CHUN, R. Y. S. Comunicação suplementar e/ou alternativa: abrangência e peculiaridades dos termos e conceitos em uso no Brasil. Pró-Fono Revista de Atualização Científica, Barueri, v. 21, n. 1, p. 69-74, 2009. DOI: https://doi.org/10.1590/S0104-56872009000100012

CORDEIRO A. M. et al. Revisão sistemática: Uma revisão narrativa. Rev Col Bras Cir., Rio de Janeiro, v. 34, n. 6, 2007. DOI: https://doi.org/10.1590/S0100-69912007000600012

DGE MEC Decreto-Lei n. 54/2018. Disponível em: http://www.dge.mec.pt/sites/default/files/EEspecial/dl_54_2018.pdf. Acesso em: 10 maio 2021.

DN/PT, Diário de Notícias de Portugal 2020. Disponível em: https://www.dn.pt/poder/costa-anuncia-que-medidas-vao-apertar-por-causa-da-pascoa12014578.html. Acesso em: 10 maio 2021.

MARTINS, H. M. L. E. P. O Museu Nacional de Arte Antiga, o edifício e a sua história: contributos para um projeto de comunicação. 2014. 153 f. Dissertação (Mestrado em Museologia) - Faculdade de Ciências Sociais e Humanas, Universidade Nova de Lisboa, Lisboa, 2014.

MARTINS, M. F. M. Estudos de Revisão de Literatura. Rio de Janeiro, 10 set. 2018. Disponível em: https://www.arca.fiocruz.br/bitstream/icict/29213/2/Estudos_revisao.pdf. Acesso em: 10 maio 2021.

MERRIAM, S. B. Qualitative research and case study applications in education. San Francisco: Jossey-Bass, 1998.

NUNES, C.; MADUREIRA, I. Desenho Universal para a Aprendizagem: Construindo práticas pedagógicas inclusivas. Revista Da Investigação às Práticas, v. 5, n. 2, p. 126-143, 2015. Disponível em: https://repositorio.ipl.pt/bitstream/10400.21/5211/1/84-172-1-SM.pdf. Acesso em: 10 maio 2021.

OMS. Organização Mundial da Saúde. Guia Considerações psicossociais e de saúde mental durante o surto de COVID-19. 2020. Disponível em:

https://www.paho.org/pt/documentos/consideraciones-psicosociales-salud-mental-durantebrote-covid-19. Acesso em: 10 abr. 2020.

ONU. Organização das Nações Unidas. A Declaração Universal dos Direitos Humanos. 1948. Disponível em: https://nacoesunidas.org/direitoshumanos/declaracao/. Acesso em: 10 maio 2020.

ONU. Organização das Nações Unidas. Convenção sobre os Direitos das Pessoas com Deficiência. 2008. Disponível em: http://www.inr.pt/content/1/1187/convencao-sobre-osdireitos-das-pessoas-com-deficiencia. Acesso em: 10 maio 2021.

RIBEIRO, G. R. P. S.; AMATO, C. A. L. H. Análise da utilização do Desenho Universal para Aprendizagem. Cadernos de Pós-Graduação em Distúrbios do Desenvolvimento, São Paulo, v. 18, n. 2, p. 125-151, jul./dez. 2018. Disponível em: http://dx.doi.org/10.5935/cadernosdisturbios.v18n2p125-151. Acesso em: 10 maio 2021. 
SANTOS, B. S. A Cruel Pedagogia do Vírus. Biblioteca Nacional de Portugal - CDU 347 Edições Almedina, S.A., 2020. ISBN 978-972-40-8496-1. Disponível em: http://www.cidadessaudaveis.org.br/cepedoc/wp-content/uploads/2020/04/Livro-BoaventuraA-pedagogia-do-virus.pdf. Acesso em: 10 jun. 2020.

SILVA, A. A. M. Sobre a possibilidade de interrupção da epidemia pelo coronavírus (COVID-19) com base nas melhores evidências científicas disponíveis. Revista Brasileira de Epidemiologia, v. 23, e200021, 2020. Disponível em:

https://www.scielo.br/pdf/rbepid/v23/1980-5497-rbepid-23 e200021.pdf. Acesso em: 10 abr. 2020.

SOUSA, C. M. A. O. A. E se entrasse numa livraria e pedisse um livro multiformato? In: ENCONTRO SOBRE INCLUSÃO EM CONTEXTO ESCOLAR, 3., 2018. Anais [...]. IPL, 2018

SOUSA, C. M. A. O. A. O conhecimento que os professores manifestam sobre a metacognição da comunicação não-verbal na escola inclusiva: respostas aos alunos com NEE. 2012. Tese (Doutoral en Educación) - Departamento de Ciencias de la Educacion, Universidad de Extremadura, Espanha, 2012. Disponível em:

http://dehesa.unex.es/xmlui/handle/10662/437. Acesso em: 10 abr. 2020.

UNESCO. Policy Guidelines on Inclusion in Education. Paris: UNESCO, 2009. Disponível em: https://www.european-agency.org/sites/default/files/key-principles-for-promotingquality-in-inclusive-education_key-principles-PT.pdf. Acesso em: 10 abr. 2020.

VERZONI, L. D. N. Sistemas Suplementares e/ou Alternativos de Comunicação (SSAC). 1999. Disponível em: http://www.profala.com/artpc5.htm. Acesso em: 10 jun. 2020.

\section{Como referenciar este artigo}

CARDOSO, E.; CASTElini, A. L. O.; SOUSA, C. M. A. O. A. Práticas inclusivas em contexto: ações de comunicação acessível em Portugal e no Brasil. Revista Ibero-Americana de Estudos em Educação, Araraquara, v. 16, n. esp. 4, p. 2990-3004, dez. 2021. e-ISSN: 1982-5587. DOI: https://doi.org/10.21723/riaee.v16iesp.4.16063

Submetido em: 07/09/2021

Revisões requeridas em: 30/10/2021

Aprovado em: 10/12/2021

Publicado em: 30/12/2021 